\title{
Nachruf auf Neil J. Smelser (1930-2017)
}

\author{
Hans-Peter Müller
}

Online publiziert: 22. Dezember 2017

(C) Springer Fachmedien Wiesbaden GmbH, ein Teil von Springer Nature 2017

Neil J. Smelser war ein großer Soziologe und ein großartiger Mann. Knapp, klar und analytisch präzise sind alle seine soziologischen Werke; diplomatisch, freundlich, hilfreich und optimistisch war er als Mensch, Kollege und Freund.

Geboren in dem winzigen Örtchen Kahoka, Missouri, das auch heute nicht viel mehr als 2000 Einwohner zählt, und aufgewachsen in Phoenix, Arizona, war er ein typischer ,country man“, der die Natur von Utah und Arizona mit ihren Canyons zeitlebens liebte. Diese ländliche Herkunft dürfte ihn wohl stets vor der sterilen Eitelkeit und dem urbanen Narzissmus bewahrt haben, den sogenannte „big shots“ nebst eigener Mythenbildung gern an den Tag legen. Trotzdem sollte er schon rasch als „Harvard and Oxford wunderkind“ (Arlie Hochschild) seinen Weg gehen. Denn Neil gewann mit 18 ein Stipendium für Harvard, und obgleich extrem erfolgreich, konnte er sich mit der elitistischen „East Coast culture“ nicht recht anfreunden, wie er in dem Interview „How Rhodes scholars think“ einräumte: „It was the only thing about Harvard that alienated me at all, because otherwise I was treated extremely well there. I was very successful academically. I made friends there. I have a loyalty to Harvard as an institution. But the emphasis on equality, plain speaking, and openness seemed to be strong and admirable features in my family. I'm not exactly sure that is wisdom, but it's something that I've always found to be a virtue on my part and I'm very proud of having that outlook on life. " ${ }^{1}$ So machte er 1952 seinen B.A. in „Social Relations“ am Harvard College, um dann mit einem Rhodes Scholarship an die Oxford University zu wechseln. 1954 folgte sein M.A. in PPE, wie es dort einfach heißt, also „Philosophy, Politics \& Economics“. Zurück in den USA legte

\footnotetext{
H.-P. Müller $(\bowtie)$

Berlin, Deutschland

E-Mail: hpmueller@sowi.hu-berlin.de
}

${ }^{1}$ https://rhodesscholars.wordpress.com/2009/11/23/neil-j-smelser. Zugegriffen: Nov. 2017. 
er 1958 seinen Ph.D. an der Harvard University ab und veröffentlichte 1959 seine Dissertation unter dem Buchtitel Social Change in the Industrial Revolution. Überdies hatte das „Wunderkind“ da schon zusammen mit Talcott Parsons Economy and Society (1956) publiziert. Beide Werke sollten ihn schlagartig bekannt machen, sodass er 1958 an die University of California in Berkeley berufen wurde, wo er recht bald (1962) zum „Full Professor“ aufrückte und bis 1994 als „University Professor“ lehrte. Zwischenzeitlich studierte er am San Francisco Psychoanalytic Institute, wo er 1971 das Examen zum Psychoanalytiker bestand, um von da ab bis ins hohe Alter ein Mal wöchentlich in San Francisco zu praktizieren. Nach seiner Emeritierung in Berkeley folgte die Station als Direktor des „Center for Advanced Study in the Behavioral Sciences“ an der Stanford University in Palo Alto bis 2001.

Ein Blick zurück auf seine beiden ersten Bücher (von insgesamt 50 - 30 eigene und 20 herausgegebene Bände!) zeigt bereits die Charakteristika von Smelsers soziologischer Denkweise: Erstens hegte er eine starke theoretische Neigung, die aufgrund der Prägung durch Talcott Parsons vielleicht nicht gänzlich überrascht. Doch bereits von Anfang an setzte er sich auch mit den Problemen und Schwächen des Strukturfunktionalismus auseinander - er an der Westküste und Robert K. Merton an der Ostküste. Statt Stabilität und soziale Ordnung zu untersuchen, studierte er sozialen Wandel und soziale Bewegungen. Statt Ordnung und Wandel über abstrakte Konzepte wie soziale Kontrolle und Sozialisation zu erfassen, studierte er historisch genauestens die jeweiligen Mechanismen des sozialen Wandels. Statt Handlung und System zu stipulieren, untersuchte er kollektives Handeln und institutionelle Bereiche wie die Ökonomie, Bildung und Familie in einer historisch-empirischen, nicht allein abstrakt-analytischen Weise. Eine Reihe seiner Werke sind in den jeweiligen Feldern zu Klassikern geworden: Social Change in the Industrial Revolution (1959) für die Differenzierungstheorie; die Theory of Collective Behavior (1962) für die soziale Bewegungsforschung; The Sociology of Economic Life $(1962,1975)$ und sein berühmtes Handbook of Economic Sociology (mit Richard Swedberg, 1994) für die Wirtschaftssoziologie, die erst nach Erscheinen dieser beiden Bücher zur vollen Blüte in den USA und in Europa gelangen sollte; sowie Social Paralysis and Social Change (1991) für die Erforschung des sozialen Wandels.

Zweitens zeigte er ein großes Interesse für methodologische Fragen der Sozialwissenschaften. In postmodernen Zeiten der „Studies“-Programme, die Intuition, Differenz und Diversität betonen, mag dieses Interesse hoffnungslos veraltet erscheinen. Dennoch dürften soziologische Analysen auch heute noch von seinen Überlegungen zu den historisch-komparativen Methoden profitieren. So etwa von seinen Comparative Methods in the Social Sciences (1976), die aus meiner Sicht zu den besten Werken auf diesem Feld zählen - in den 1980er-Jahren machten wir in Heidelberg mit dem Max-Weber-Gastprofessor Reinhard Bendix (Berkeley), M. Rainer Lepsius und Wolfgang Schluchter ein Seminar über historisch-komparative Methoden in den Sozialwissenschaften, in dem wir Bendix, Barrington Moore, Victoria Bonnell, Theda Skocpol und natürlich Neil Smelser lasen. Auch während seiner Georg-SimmelGastprofessur an der Humboldt-Universität zu Berlin (nach dem Inauguralprofessor Lewis A. Coser und nach M. Rainer Lepsius) im Jahre 1995 machte er sich Gedanken über die methodologischen Aggregatsebenen soziologischer Analysen. In 
Problematics of Sociology (1997) behandelte Smelser in vier instruktiven Kapiteln die Mikro-, Meso- und Makrosoziologie sowie die globale Soziologie.

Ein weiterer Grundzug seiner Denkweise wird deutlich, wenn man die gesellschaftlichen Felder betrachtet, die Smelser untersucht hat: Erstens, die Ökonomie und ihre Hegemonie, der Smelser mit einer Wirtschaftssoziologie zu begegnen suchte. Zweitens, das Bildungssystem und die Rolle der Universitäten in den westlichen Gesellschaften. Man lese noch einmal den spannenden Epilog in Talcott Parsons' und Gerald Platts The American University (1973), seine Überlegungen zu The Changing Academic Market (1980), seine Reflections on the University of California: From the Free Speech Movement to the Global University (2010) oder sein Buch Dynamics of the Contemporary University: Growth, Accretion, and Conflict (2013), um zu ermessen, wie sehr ihm das Schicksal und die Zukunft der Universität am Herzen lagen. Drittens, seine soziologischen und psychoanalytischen Beiträge zum Verständnis der Familie - sein mit Erik H. Erikson herausgegebenes Buch Themes of Work and Love in Adulthood (1980), sein historisches Porträt The Victorian Family (1982) oder sein mit Sydney Halpern verfasster Essay The Historical Triangulation of Family, Economy, and Education (1978).

Aber Smelser war eben nicht nur ein Soziologe, der viel geschrieben oder hohe Drittmittel eingeworben hat. Er betätigte sich auch als effektiver Manager und begnadeter Mediator sowie als Institutionenbildner - wohl ein typisches Phänomen dieser Generation. Nicht so sehr der eigene individuelle Erfolg, sondern das Wohlergehen der Institution Universität und seiner Bewohner, den Studenten, war es, was diese Altersgruppe - zu der in Deutschland Ralf Dahrendorf, M. Rainer Lepsius oder Friedhelm Neidhardt gehören - vornehmlich umgetrieben hat. Als die Soziologie an der Harvard University in eine ernsthafte Krise geraten war, half Smelser, ein neues Department zu begründen. Er war Vizepräsident der „International Sociological Association“ und 88. Präsident der „American Sociological Association“ (1997). Er initiierte mit Jeffrey C. Alexander, Bernhard Giesen und Richard Münch die berühmten amerikanisch-deutschen Theoriekonferenzen in den 1980er-Jahren, aus denen u.a. der einschlägige Band The Micro-Macro Link (1987) hervorging.

Auch nach seinem Ausscheiden aus dem institutionellen akademischen Leben war Smelser von begnadeter Produktivität und unerschöpflicher Neugier. Als Gastprofessor in Berkeley 2009 war ich Zeuge, wie er gerade nach Beendigung seiner Bücher über den Terrorismus und die Odyssee des Lebens an seinem soziologischen Vermächtnis schrieb, um das konzentrierte Schreiben aber jederzeit bereitwillig zu unterbrechen, um unsere unmöblierte Gästewohnung auf dem Campus mit eigenen Möbelstücken aus seinem großen Haus auszustatten. Aber nicht The Faces of Terrorism (2007) und auch nicht mein Lieblingsbuch The Odyssey Experience (2009) bezeichnete er als sein geistiges Erbe, sondern die Usable Social Science (2012, mit John S. Reed). Ein Soziologe und ein Banker mit dem Selbstverständnis eines Bankiers (Reed war viele Jahre CEO der Citybank) hatten sich zusammengesetzt, um das positive Wissen der Soziologie zusammenzutragen. Reed war überzeugt, dass die Finanzkrise von 2008 nicht ausgebrochen wäre, wenn amerikanische Manager über ein solides soziologisches Wissen verfügt hätten. Da Manager keine Zeit haben zu lesen, sollte dieses eine Buch die Wissenslücke im amerikanischen Management füllen. Auch wenn das sicherlich eine allzu optimistische Annahme 
war, bot das Buch Neil Smelser doch die große Gelegenheit, das gesamte Wissen aus seinem soziologischen Leben zu komprimieren und analytisch wie empirisch so $\mathrm{zu}$ verdichten, dass man diesem Wissensangebot sogar so etwas wie eine Rezeptur für dilemmatische Entscheidungssituationen entnehmen konnte. Reed selbst steuerte Zwischenbetrachtungen mit Fallbeispielen bei, die genau solche dramatischen Entscheidungssituationen aus seinem Arbeitsleben bei der Citybank schilderten. Usable Social Science kann man getrost nicht nur Managern, sondern auch Soziologen für ihren Nachttisch empfehlen.

Mit Neil Smelser verliert nicht nur die amerikanische, sondern auch die internationale Soziologie einen der ganz Großen ihres Faches. Der einzige Trost ist, dass er durch sein Werk weiterleben wird - und so im kollektiven Gedächtnis der soziologischen Zunft eine wichtige Bezugsgröße werden kann. Wer keine Zeit mehr zum Lesen hat, kann einen nachdenklichen und aufgeräumten Neil Joseph Smelser in der legendären Berkeley-Sendung von Harry Kreisler „Conversations with History“ vom August 2010 auch visuell nacherleben. ${ }^{2}$

2 https://conversations.berkeley.edu/content/neil-j-smelser. Zugegriffen: Nov. 2017. 